Proc. Indian Acad. Sci. (Math. Sci.) Vol. 115, No. 4, November 2003, pp. 429-435.

Printed in India

\title{
Transversals in non-discrete groups
}

\author{
RAMJI LAL and R P SHUKLA \\ Department of Mathematics, University of Allahabad, Allahabad 211 002, India \\ E-mail: ramjilal@mri.ernet.in; rps@mri.ernet.in
}

MS received 2 August 2004; revised 4 August 2005

\begin{abstract}
The concept of 'topological right transversal' is introduced to study right transversals in topological groups. Given any right quasigroup $S$ with a Tychonoff topology $T$, it is proved that there exists a Hausdorff topological group in which $S$ can be embedded algebraically and topologically as a right transversal of a subgroup (not necessarily closed). It is also proved that if a topological right transversal $\left(S, T_{S}, T^{S}, \circ\right)$ is such that $T_{S}=T^{S}$ is a locally compact Hausdorff topology on $S$, then $S$ can be embedded as a right transversal of a closed subgroup in a Hausdorff topological group which is universal in some sense.
\end{abstract}

Keywords. Topological transversal; Hilbert space; free topological group.

\section{Introduction}

The importance of the study of transversals in groups individually and abstractly has been explained in [7], [8], and [12]. Every right transversal of a subgroup in a group has the structure of a right quasigroup with identity. Conversely, every right quasigroup with identity can be embedded as a right transversal into a group which is universal in some sense (see Theorem 3.4 of [7]). Transversals in a group have more control on the structure of the group than subgroups. For example, there are uncountably many non-Dedekind (2-generator infinite simple groups (Theorem 28.7 of [10]) and (Theorem 28.8 of [10])) groups all of whose non-trivial proper subgroups are isomorphic, whereas there is no nonDedekind group with all non-trivial proper transversals isomorphic (as right quasigroups) [89].

The abstract description of right transversals of a closed subgroup in a topological group is a difficult problem and needs more serious thought. In this paper, we make an attempt in this direction.

\section{Right transversals in topological groups}

Let $S$ be a right transversal of a closed subgroup $H$ in a Hausdorff topological group $G$. Algebraically, $S$ is a right quasigroup with identity (the right quasigroup structure $\circ$ on $S$ is defined by $\{x \circ y\}=H x y \cap S)$. Let $\langle S\rangle$ denote the subgroup of $G$ generated by $S$ and $H_{S}$ denote the subgroup $\langle S\rangle \cap H$. Then $H_{S}=\left\langle\left\{x y(x \circ y)^{-1}: x, y \in S\right\}\right\rangle$, and $H_{S} S=\langle S\rangle$, where $\circ$ is defined as above. Identifying $S$ with the set $H \backslash G$ of right cosets of $H$ in $G$, we obtain a permutation representation $\phi: G \rightarrow \operatorname{Sym}(S)(\{\phi(g)(x)\}=S \cap H x g, g \in G, x \in S$ and we adopt the convention that $(r . s)(x)=s(r(x)), r, s \in \operatorname{Sym}(S)(x \in S)$. Let $G_{S}=\phi\left(H_{S}\right)$. Then 
$G_{S}$ depends only on the right quasigroup structure $\circ$ on $S$ and not on the subgroup $H$ (see [7]). This group is called the group torsion of the right quasigroup $(S, \circ)$ [7]. It is easy to observe that $\phi$ is injective on $S$ and if we identify $S$ with $\phi(S)$, then $\phi(\langle S\rangle)=G_{S} S$ is a group which also depends only on the right quasigroup $(S, \circ)$ and in which $S$ is a right transversal of $G_{S}$. This group has some universal property [7] and is called the group extension of $(S, \circ)$. Also $\langle S\rangle=H_{S} S$ is a topological subgroup of $G$ and $G_{S} S$ has quotient topological group structure in which $G_{S}$ is closed (for $H_{S}=\phi^{-1}\left(G_{S}\right)=H \cap\langle S\rangle$ is closed in $\langle S\rangle$ ) and $S$ is a right transversal of $G_{S}$ in $G_{S} S$. We have two Tychonoff topologies on $S$, viz. $T_{S}$, the subspace topology on $S$ induced by the topology of $G_{S} S$ and $T^{S}$, the quotient topology on $S$ induced by the natural projection from $G_{S} S$ to $S$. Clearly $T_{S}$ is finer than $T^{S}$. Let $T_{G_{S}}$ denote the subspace topology on $G_{S}$ (with respect to which it is a topological group) and $T^{G_{S}}$, the quotient topology on $G_{S}$ induced by the natural projection from $G_{S} S$ to $G_{S}$. The topology $T^{G_{S}}$ need not be even a $T_{1}$ topology (Example 2.3). Since $(S, \circ)$ is a right quasigroup with identity, we have another map $\chi: S \times S \rightarrow S$ given by

$$
\chi(x, y) \circ x=y .
$$

The binary operations $\circ$ (and also $\chi$ ) may not be continuous with respect to either of the topologies $T_{S}$ and $T^{S}$ on $S$. For example take $G=S O(n)$ and $H=S O(n-1)$ where $n \notin$ $\{1,2,4,8\}$. The quotient space $H \backslash G$ is homeomorphic to $S^{n-1}$. There is a right transversal $S$ of $H$ in $G$ (see Example 2.3) such that $G_{S}=H$ and $G_{S} S=G$. Then $\left(S, T^{S}\right)$ is homeomorphic to $S^{n-1}$ with the usual topology. Since there is no continuous right (left) quasigroup structure on $S^{n-1}$ for $n \notin\{1,2,4,8\}$ [1], o and $\chi$ are not continuous from $\left(S \times S, T^{S} \times T^{S}\right)$ to $\left(S, T^{S}\right)$, where $\left(S \times S, T^{S} \times T^{S}\right)$ is the product of $\left(S, T^{S}\right)$ with itself. Next, there is a choice of a right transversal of $S$ in $G$ (see Example 2.3 with $\mathbf{H}=\mathbb{R}^{n}$ ) for which $T_{S}$ is non-homogeneous, and as such $\circ:\left(S \times S, T_{S} \times T_{S}\right) \rightarrow\left(S, T_{S}\right)$ is not continuous. However, $\circ$ and $\chi$ are continuous from $\left(S \times S, T_{S} \times T_{S}\right)$ to $\left(S, T^{S}\right)$.

The above discussion prompts us to have the following definition.

\section{DEFINITION 2.1.}

A quadruple $\left(S, T_{S}, T^{S}, \circ\right)$, where $(S, \circ)$ is a right quasigroup with identity, $T_{S}$ and $T^{S}$ are two Tychonoff topologies with $T_{S} \supseteq T^{S}$ and $T^{S}$ homogeneous is called a topological right transversal if

(i) $\circ$ is continuous map from $\left(S \times S, T_{S} \times T_{S}\right)$ to $\left(S, T^{S}\right)$, and also

(ii) the map $\chi:\left(S \times S, T_{S} \times T_{S}\right) \rightarrow\left(S, T^{S}\right)$ defined by the equation $\chi(x, y) \circ x=y$, is continuous.

We shall say that a topological right transversal $\left(S, T_{S}, T^{S}, \circ\right)$ is proper if $(S, \circ)$ is not a group. This terminology is justified (for such a right transversal in any group is always a proper transversal). Every choice of a right transversal of a closed subgroup $H$ of a topological group $G$ determines a topological right transversal. Trivially, an improper topological transversal $\left(G, T_{G}, T^{G}, \circ\right)$, where $T_{G}$ is a discrete topology on $G$ and $T^{G}$ is any non-discrete homogeneous Tychonoff topology cannot arise as a right transversal in the above manner. Indeed, it comes from a topological right transversal if and only if $T_{G}=T^{G}$.

The following example says that even a proper topological right transversal need not arise as a right transversal of a closed subgroup in a topological group. 
Example 2.2. For each sufficiently large odd $n\left(n>10^{10}\right)$ and $m>1$, there is a central extension $K$ of $B(m, n)$ (the Burnside group of exponent $n$ on a set containing $m$ elements) by a cyclic group $C_{n}$ of order $n$ which admits no non-discrete topology (Theorem 31.5 of [10]). Take $G=K \times D$, where $D$ is a finite group having a non-normal subgroup $H$ of prime order $p$. Let $T$ be a topology on $G$ with respect to which it is a $T_{1}$-topological group. As observed earlier, the induced subspace topology on $K \times\{e\}$ is the discrete topology (where $e$ is the identity element in $D$ ). Since every discrete subgroup of a $T_{1}$ topological group is closed (Theorem 5.10 of [6]), $K \times\{e\}$ is a closed subgroup of $G$. But, then $K \times\{d\}$ is closed for each $d \in D$. Since $D$ is finite, this implies $K \times\{d\}$ is open for each $d \in D$. Since $K \times\{d\}$ is open and discrete for all $d \in D$, it follows that $T$ is the discrete topology. This shows that $G$ also does not admit any non-discrete $T_{1}$-topology. Let $H$ be a non-normal subgroup of $D$ of order $p$. Then there is a right transversal $S$ of $\{e\} \times H$ in $G$ which generates $G$ and $G_{S} S \cong G$ (see Proposition 3.10 of [7]). Thus $G_{S} S$ admits only discrete topology. Hence $\left(S, T_{S}, T^{S}, \circ\right)$, where $\circ$ is the binary operation induced on $S$ with respect to which it is a right quasigroup, and arises from a topological group structure on $G_{S} S$ if and only if $T_{S}=T^{S}$ is the discrete topology. Take any non-discrete homogeneous Tychonoff topology $T^{S}$ on $S$ (note that such a topology exists, viz. the topology induced by the topology of $Q$ (by fixing a bijection between $S$ and $Q)$ ), then $\left(S, T_{S}, T^{S}, \circ\right.$ ), where $T_{S}$ is the discrete topology on $S$, is a topological right transversal. Observe that $\left(S, T_{S}, T^{S}, \circ\right)$ will not arise from any topological group structure on $G_{S} S$ (note that $\left(S, T_{S}, T^{S}, \circ\right)$ is a proper transversal).

Example 2.3. Transversal structures on spheres in Hilbert spaces. Let $\mathbf{H}$ be a real Hilbert space with $\langle$,$\rangle as inner product structure. Let O(\mathbf{H})$ denote the topological group of isometries of $\mathbf{H}$. For the product in $O(\mathbf{H})$, we adopt the convention that $\left(T_{1} T_{2}\right)(x)=T_{2}\left(T_{1}(x)\right)$; $T_{1}, T_{2} \in O(\mathbf{H})$ and $x \in \mathbf{H}$. Let $S^{\mathbf{H}}=\{x \in \mathbf{H}:\|x\|=1\}$ denote the unit sphere in $\mathbf{H}$ (in the case of $\mathbf{H}=\mathbb{R}^{n}$, we denote it by $S^{n-1}$ ). Fix a point $e_{0} \in S^{\mathbf{H}}$. Let $V=\left\langle e_{0}\right\rangle^{\perp}$ (the orthogonal complement of the subspace $\left\langle e_{0}\right\rangle$ generated by $\left.e_{0}\right)$ and $O(V)=\left\{T \in O(\mathbf{H}): T\left(e_{0}\right)=e_{0}\right\}$. Then $O(V)$ is a closed subgroup of $O(\mathbf{H})$. The topological group $O(\mathbf{H})$ acts transitively on $S^{\mathbf{H}}$ from right and the isotropy group at $e_{0}$ is $O(\mathbf{H})_{e_{0}}=O(V)$. This gives us a bijective continuous map $\phi$ from $O(V) \backslash O(\mathbf{H})$, the quotient space of right cosets of $O(V)$ in $O(\mathbf{H})$ to $S^{\mathbf{H}}$ given by $\phi(O(V) T)=T\left(e_{0}\right)$. It is easy to observe that $\phi$ is a homeomorphism.

Let $x \in S^{\mathbf{H}}$. Define a map $R_{x}: \mathbf{H} \rightarrow \mathbf{H}$ by

$$
R_{x}= \begin{cases}J\left(2 P_{\frac{e_{0}+x}{\left\|e_{0}+x\right\|}}-I\right), & \text { if } x \neq-e_{0} \\ -I, & \text { if } x=-e_{0}\end{cases}
$$

where for $a \in \mathbf{H}, P_{a}$ denotes the projection of $\mathbf{H}$ onto $\mathbb{R} a$ and $J$ is defined by

$$
J(x)=\left\langle x, e_{0}\right\rangle e_{0}-\left(x-\left\langle x, e_{0}\right\rangle e_{0}\right)=2\left\langle x, e_{0}\right\rangle e_{0}-x
$$

Thus for any non-zero $a \in \mathbf{H}$,

$$
P_{a}(x)=\left\langle x, \frac{a}{\|a\|}\right\rangle \frac{a}{\|a\|}, \quad x \in \mathbf{H} .
$$

Clearly the map $R_{x} \in O(\mathbf{H})$. Also the map $R: S^{\mathbf{H}} \rightarrow O(\mathbf{H})$ defined by $R(x)=R_{x}$ is injective and is a set theoretic section (not continuous at $-e_{0}$ ) of the quotient map $\phi$. Thus the image 
$R\left(S^{\mathbf{H}}\right)=\left\{R_{x}: x \in S^{\mathbf{H}}\right\}$ is a right transversal of $O(V)=O(\mathbf{H})_{e_{0}}$ in $O(\mathbf{H})$. Identifying $R\left(S^{\mathbf{H}}\right)$ with $S^{\mathbf{H}}$ through the map $R$, we get a subspace topology $T_{S^{\mathbf{H}}}$ on $S^{\mathbf{H}}$. We observe that the usual subspace topology of $S^{\mathbf{H}}$ in $\mathbf{H}$ is same as the quotient topology $T^{S^{\mathbf{H}}}$ on $S^{\mathbf{H}}$ induced by the map $\phi$. This gives us a topological right transversal structure $\left(S^{\mathbf{H}}, T_{S} \mathbf{H}, T^{S^{\mathbf{H}}}, \circ\right)$ on $S^{\mathbf{H}}$. The right quasigroup structure $\circ$ on $S^{\mathbf{H}}$ is defined by

$$
x \circ y=\left\{\begin{array}{ll}
\left(2 P_{\frac{e_{0}+y}{\left\|e_{0}+y\right\|}}-I\right)(J(x)), & \text { if } y \neq-e_{0} \\
-x, & \text { if } y=-e_{0}
\end{array} .\right.
$$

Since the section $R$ is not continuous at $-e_{0}$, the topology $T_{S^{\mathrm{H}}}$ is strictly finer than $T^{S^{\mathrm{H}}}$ and is not homogeneous. In fact $\left\{-e_{0}\right\}$ is an isolated point of $\left(S^{\mathbf{H}}, T_{S^{\mathbf{H}}}\right)$ and the subspace topologies on $S^{\mathbf{H}} \backslash\left\{-e_{0}\right\}$ induced by $T_{S^{\mathbf{H}}}$ and $T^{S^{\mathbf{H}}}$ are same. Thus, $\left(S^{\mathbf{H}}, T_{S^{\mathbf{H}}}\right)$ is locally compact. It can also be seen that $O(\mathbf{H})$ is generated by $S^{\mathbf{H}}$ (more precisely $R\left(S^{\mathbf{H}}\right)$ ) and the core of $O(V)$ in $O(\mathbf{H})$ is trivial. This implies that (see Proposition 3.10 of [7]) $G_{S} S^{\mathbf{H}}=$ $O(\mathbf{H})$. We also observe that the quotient topology $T^{G_{S} \mathbf{H}}$ induced by the projection map from $G_{S^{\mathbf{H}}} S^{\mathbf{H}}$ to $G_{S \mathbf{H}}$ is not a $T_{1}$-topology (for $S^{\mathbf{H}}$ is not closed in $O(\mathbf{H})$ ).

\section{Transversals on locally compact Hausdorff spaces}

\section{DEFINITION 3.1. [2].}

Let $X$ be a locally compact Hausdorff space. Let $G=\operatorname{Homeo}(X)$ be the group of homeomorphisms of $X$. Let $K$ and $W$ be closed and open subspaces of $X$ respectively such that either $K$ is compact or the complement of $W$ in $X$ is compact. Let $(K, W)$ denote the set of all homeomorphisms of $X$ which send $K$ into $W$. The $g$-topology on $G$ is the topology generated by the set $\mathscr{S}$ as a subbase whose members are of the form $(K, W)$.

Theorem 3.2. Let $(S, T, T, \circ)$ be a topological right transversal, where $T$ is a locally compact Hausdorff topology on $S$. Then there exists a unique Hausdorff topological group $G^{S}$ containing $(S, T, T, \circ)$ as a topological right transversal such that given any topological group $G$ containing $(S, T, T, \circ)$ as a right transversal, there exists a unique continuous homomorphism from $G$ to $G^{S}$ which is the identity map on $S$.

Proof. Let $(S, T, T, \circ)$ be a topological right transversal with $T$ a locally compact Hausdorff topology on $S$. Then the binary operation $\circ$ and the map $\chi$ defined by $\chi(x, y) \circ x=y$ are continuous from $(S \times S, T \times T)$ to $(S, T) . S \backslash\{e\}$ ( $e$ is the identity of $(S, \circ))$ being open subspace of $S$ is also locally compact. Thus Homeo $(S \backslash\{e\})$ is a topological group with respect to the $g$-topology (Theorem 3 of [2]). Also the natural action $\theta: S \times \operatorname{Homeo}(S) \rightarrow S$ is continuous [3]. Hence $\theta \mid S \times H^{s}=\theta^{s}$, from $S \times H^{s}$ to $S$ is also continuous, where $H^{s}=\{f \in \operatorname{Homeo}(S): f(e)=e\}$. Then $\left(S, H^{s}, \sigma^{s}, f^{s}\right)$ is a $c$-groupoid (see Definition 2.1 and p. 78 of [7]), where $f^{s}$ is the map from $S \times S$ to $H^{s}$ defined by

$$
f^{s}(x, y)(z)=\chi(x \circ y,(z \circ x) \circ y), \quad x, y, z \in S
$$

and the map $\sigma^{s}$ from $S \times H^{s}$ to $H^{s}$ defined by $\left(\sigma^{s}(x, h)\right.$ is denoted by $\sigma_{x}^{s}(h)$ ),

$$
\sigma_{x}^{s}(h)(y)=\chi\left(x \theta^{s} h,(y \circ x) \theta^{s} h\right), \quad x, y \in S, \quad h \in H^{s} .
$$


Let $G^{S}=H^{s} S$ be the general extension of $H^{s}$ by $S$ determined by the $c$-groupoid $\left(S, H^{s}, \sigma^{s}, f^{s}\right)$ (p. 79 of [7]). The product in $G^{S}$ is given by

$$
h x \cdot k y=h \sigma_{x}^{s}(k) f^{s}\left(x \theta^{s} k, y\right) x \theta^{s} k \circ y,
$$

and the inverse of $h x$ is given by

$$
(h x)^{-1}=\left(f^{s}\left(x^{\prime}, x\right)\right)^{-1} \sigma_{x^{\prime}}^{s}\left(h^{-1}\right) x^{\prime} \theta^{s} h^{-1},
$$

where $h, k \in H^{s}, x, y \in S$ and $x^{\prime}$ is the left inverse of $x$ in $(S, \circ)$ (p. 72 of [7]). We note that the subgroup $\langle S\rangle$ of $G^{S}$ generated by $S$ is $G_{S} S$.

Now, we show that $G^{S}$ is a topological group with respect to the product topology on $H^{s} \times S$. The evaluation map $E\left(f^{s}\right):(S \times S) \times S \rightarrow S$ (defined by $E\left(f^{s}\right)((x, y), z)=$ $\left.f^{s}(x, y)(z)\right)$, being the compsosition of continuous maps $((x, y), z) \mapsto(x \circ y,(z \circ x) \circ y)$ from $(S \times S) \times S$ to $S \times S$ and $\chi$, is continuous. It follows (Theorem 3.1, p. 261 of [4]) that $f^{s}: S \times S \rightarrow H^{s}$ is a continuous map. Consider the map

$$
\Sigma: S \times H^{s} \rightarrow H^{s},(x, h) \mapsto \sigma_{x}^{s}(h) .
$$

Again the evaluation map

$$
E(\Sigma):\left(S \times H^{s}\right) \times S \rightarrow S,((x, h), y) \mapsto y \theta^{s} \sigma_{x}^{s}(h),
$$

being the composition of the continuous maps $((x, h), y) \mapsto\left(x \theta^{s} h,(y \circ x) \theta^{s} h\right)$ from $(S \times$ $\left.H^{S}\right) \times S$ to $S \times S$ and $\chi$, is continuous. It follows (Theorem 3.1, p. 261 of [4]) that $\Sigma$ is also continuous. Further, the map $x \mapsto x^{\prime}$ ( $x^{\prime}$ is the left inverse of $x$ in $S$ ) from $S$ to $S$ being the composition of continuous maps $x \mapsto(x, e)$ from $S$ to $S \times\{e\}$ and $\chi$, is continuous. Using the continuity of the above maps, we find that the binary operation in $G^{S}$ and the map $g \mapsto g^{-1}$ from $G^{S}$ to itself are continuous. Finally, we observe that induced subspace topology on $S$ in $G^{S}$ is the given topology on $S$ and induced right quasigroup structure on the right transversal $S$ of the closed subgroup $H^{s}$ in $G^{S}$ is the given right quasigroup structure.

Further, suppose that $G$ is a topological group such that $(S, T, T, \circ)$ is a topological right transversal of a closed subgroup $H$ in $G$. Then the action $\theta$ of $H$ on $S$ defined by $\{\theta(x, h)\}=S \cap H x h$, where $x \in S$ and $h \in H$, is continuous. As such it induces a continuous homomorphism $q: H \rightarrow H^{s}$. Let $\phi: G \rightarrow G^{S}$ be the map defined by $\phi(a x)=q(a) x$. Then $\phi$ is a group homomorphism (see Theorems 2.6 and 3.4 of [7|) which is the identity map on $S$. We also note that $\phi$ is continuous. The uniqueness of $\phi$ follows from Theorem 3.4 in [7].

\section{COROLLARY 3.3.}

A triple $(S, T, \circ)$, where $(S, T)$ is a compact Hausdorff space and $(S, \circ)$ a right quasigroup with identity, can be embedded as a right transversal of a closed subgroup in a Hausdorff topological group if and only if the map o and the map $\chi$ from $S \times S$ to $S$ defined by $\chi(x, y) \circ x=y$ are continuous.

Proof. Since a compact Hausdorff space is a minimal Tychonoff space, $\left(S, T, T^{\prime}, \circ\right)$ is a topological right transversal if and only if $T=T^{\prime}$.

\section{COROLLARY 3.4.}

$S^{n}=\left\{x \in \mathbb{R}^{n}:\|x\|=1\right\}$ can be embedded (topologically) as a right transversal of a closed subgroup in a Hausdorff topological group if and only if $n=0,1,3,7$. 
Proof. Suppose that $S^{n}$ is embedded as a right transversal of a closed subgroup in a topological group $G$. Then by Corollary 3.3, there exists a continuous binary operation $\circ$ on $S^{n}$ such that $\left(S^{n}, \circ\right)$ is a right quasigroup with identity and the map $\chi: S^{n} \times S^{n} \rightarrow S^{n}$ given by $\chi((x, y)) \circ x=y$ is continuous. By a well-known result due to Adams [1], $n=0,1,3$ or 7 . Conversely $S^{0}, S^{1}$ and $S^{3}$ are Lie groups with respect to real, complex and quarternionic multiplication respectively. Let $\circ$ denote the Cayley multiplication on $S^{7}$. Then $\circ$ is continuous such that $\left(S^{7}, 0\right)$ is a right quasigroup with identity. Further the map $\chi: S^{7} \times S^{7} \rightarrow S^{7}$ satisfying $\chi((x, y)) \circ x=y$ is given by $\chi((x, y))=y \circ x^{-1}$ (note that Cayley multiplication satisfies $\left(x \circ y^{-1}\right) \circ y=x \circ\left(y^{-1} \circ y\right)=x$ and $(y \circ z)^{-1}=z^{-1} \circ y^{-1}$ although the multiplication is not associative) and so $\chi$ is also continuous. The result now follows from Corollary 3.3.

The following example shows that there exists a proper right transversal which does not admit any non-discrete locally compact Hausdorff topology.

Example 3.5. Let $K$ be an uncountable group which does not admit any non-discrete $T_{1}$ topology making $K$ a topological group (for existence, see p. 339 of [10] and [11]). Take $G=K \times D$, where $D$ is a finite group having a non-normal subgroup $H$ of prime order $p$. Let $T$ be a topology on $G$ with respect to which it is a $T_{1}$-topological group. Then as argued in Example 2.2, $T$ is the discrete topology on $G$. Let $H$ be a non-normal subgroup of $D$ of order $p$. Then $\{e\} \times H$ is a non-normal subgroup of $G$ of order $p$. Let $S$ be a transversal of $\{e\} \times H$ in $G$ which generates $G$. Then $G_{S} S \cong G$. Thus $G_{S} S$ admits only discrete topology. Now, if $S$ admits a non-discrete locally compact Hausdorff topology, then by Theorem 3.2, we get non-discrete locally compact Hausdorff topology on $G=G_{S} S$.

\section{Transversals of subgroups (not necessarily closed)}

This section is devoted to the following problem.

Problem 4.1. Consider a triple $(S, T, \circ)$, where $(S, T)$ is an infinite Tychonoff space and $(S, \circ)$ a right quasigroup with identity. Can we embedd $(S, \circ)$ as a right transversal of a subgroup in a topological group $G$ so that the subspace topology on $S$ is $T$ ?

The answer to the above problem is in negative if we stick to the right transversals of closed subgroups (see Example 4.3). The following theorem answers the above problem affirmatively, provided we are satisfied with the right transversal of a subgroup (not necessarily closed) in a Hausdorff topological group.

Theorem 4.2. Let $(S, T)$ be a Tychonoff space. Then for any right quasigroup structure on $S$ with e as the identity element, $S$ becomes a right transversal to a subgroup $H$ (not necessarily closed) in a Hausdorff topological group $G$ such that (i) the induced right quasigroup structure on $S$ is the given right quasigroup structure and (ii) the subspace topology induced on $S$ in $G$ is the given topology $T$ and $S$ is closed in $G$.

Proof. Let $(S, \circ)$ be a right quasigroup with $e$ as the identity. Let $G=F(S \backslash\{e\})$ be the free topological group on the subspace $S \backslash\{e\}$ of $S$ [5]. Algebraically, $F(S \backslash\{e\})$ is simply a free group on $S \backslash\{e\}$ [5]. We also know [5] that $S \backslash\{e\}$ is a closed subspace of $G$. Identifying $1 \in G$ with $e \in S$, it is possible to introduce a topology $T$ on $G$ which induces the given topology on $S$ and in which $S$ is closed [5]. Let $G_{S} S$ be the group extension 
of the right quasigroup $S$. Since $G_{S} S$ is generated by $S \backslash\{e\}$, there is a unique surjective homomorphism $\eta$ (say) from $G$ to $G_{S} S$ whose restriction on $S$ is the identity map on $S$. Thus $G_{S} S$ is a topological group with respect to the quotient topology (induced by $\eta$ ) which need not be Hausdorff. The topology on $S$ induced by the topology of $G_{S} S$ may be strictly coarser than the topology $T$ on $S$ (see Example 2.3). However, $S$ can also be considered as a right transversal of a subgroup $H=\eta^{-1}\left(G_{S}\right)$ in $G$. If $g \in G, g \neq 1$, then $\eta(g)=h x$ for some $h \in G_{S}$ and $x \in S$. Since $\eta$ is surjective, there is $k \in H$ such that $\eta(k)=$ $h$ and then $\eta(g)=h x=\eta(k) \eta(x)=\eta(k x)$. This shows that $g=k^{\prime} x$ for some $k^{\prime} \in H$. Also, if $g=k_{1} x_{1}=k_{2} x_{2}$, where $k_{1}, k_{2} \in H$ and $x_{1}, x_{2} \in S$, then $\eta(g)=\eta\left(k_{1}\right) x_{1}=\eta\left(k_{2}\right) x_{2}$. This means $x_{1}=x_{2}$ and hence $k_{1}=k_{2}$. One can also observe that the right quasigroup structure induced on the right transversal $S$ of $H$ in $G$ is the same as the right quasigroup structure on $S$ from which we started.

Example 4.3. Consider the topological right transversal $S$ discussed in Example 2.2. Let $T$ be any non-discrete homogeneous Tychonoff topology. Then as in the notation of the above theorem, $H=\eta^{-1}\left(G_{S}\right)$ is not closed as otherwise we get a non-discrete $T_{1}$ topological group structure on $G_{S} S$ in which $S$ becomes a topological right transversal of the closed subgroup $G_{S}$. This contradicts Example 2.2.

\section{Acknowledgement}

We would like to thank the referee for his/her valuable comments and suggestions.

\section{References}

[1] Adams J F, On the non-existence of Hopf invariant one, Ann. Math. 72(1) (1960) 20-104

[2] Arens R, Topologies for homeomorphism groups, Am. J. Math. 68 (1946) 593-610

[3] Dieck T T, Transformation groups (Berlin: Walter de Gruyter) (1987)

[4] Dugundji J, Topology (New Delhi: Prentice Hall of India) (1975)

[5] Graev M I, On free topological groups, in: Topology and topological algebra (translation, A.M.S.) (1962) vol. 8, series 1, pp. 305-364

[6] Hewitt E and Ross K A, Abstract harmonic analysis I (Berlin: Springer-Verlag) (1963)

[7] Lal R, Transversals in groups, J. Alg. 181 (1996) 70-81

[8] Lal R and Shukla R P, Perfectly stable subgroups of finite groups, Comm. Alg. 24(2) (1996) 643-657

[9] Lal R and Shukla R P, A characterization of Tarski monsters, submitted

[10] Ol'shanskii A Yu, Geometry of defining relations in groups (London: Kluwer Academic Publishers) (1991)

[11] Shelah S, On a problem of Kurosh, Jonsson group, and applications, in: Word problems II (Amsterdam: North-Holland) (1980) pp. 373-394

[12] Shukla R P, Congruences in right quasigroups and general extensions, Comm. Alg. 23(7) (1995) 2679-2695 